\title{
An angle-distribution effect in mental rotation
}

\author{
CHARLES E. COLLYER \\ University of Rhode Island, Kingston, Rhode Island
}

\begin{abstract}
Rossi and Collyer (1986) reported data suggesting a threshold for mental rotation. The thresholdlike nonlinearity may have been caused by the skewed distribution of angles used in their mental rotation experiment. In the present study, skewing the angle distribution toward small angles (skewed condition) increased the overall slope of the mental rotation response time function, relative to that of an even-distribution condition. Visual inspection of the data suggests that if there is a threshold-like nonlinearity, it is present in both the skewed and the even conditions. However, it is difficult to draw a strong conclusion for logical and statistical reasons.
\end{abstract}

Recently, Rossi and Collyer (1986) reported a mental rotation experiment that yielded an unusual response time function. In addition to the large and approximately linear effect of angular disparity on response time, there seemed to be a flat step in the function from $0^{\circ}$ to about $15^{\circ}-20^{\circ}$. One interpretation of such a nonlinearity is the idea of a threshold for mental rotation; according to this idea, the time-consuming process of mental rotation would not be necessary for small angular disparities.

Is there a threshold for mental rotation? It is still difficult to say. A four-parameter threshold model was found to fit the Rossi and Collyer (1986) data better than a straight line, and it gave a threshold estimate of about $20^{\circ}$. However, the threshold model's better fit should be interpreted cautiously because of its larger number of free parameters. It is possible that the apparent threshold is an artifact of capitalization on chance. Collyer (1985) noted this possibility in a methodological paper on the comparison of strong and weak models, using the Rossi and Collyer data as an illustration.

Unlike most experiments on mental rotation, the Rossi and Collyer (1986) experiment used a skewed distribution of angular disparities. Most experiments have used an even spacing of disparities from $0^{\circ}$ to $180^{\circ}$, at intervals of $20^{\circ}$ or $30^{\circ}$. Rossi and Collyer used disparities spaced at intervals of $3^{\circ}$ from $0^{\circ}$ to $30^{\circ}$, and at intervals of $30^{\circ}$ from $30^{\circ}$ to $180^{\circ}$. The purpose of the heavier sampling of small angles was to provide higher resolution in the measurement of a threshold. However, it is possible that the skewed distribution itself induced changes in the shape of the response time function. Effects of angle distribution on reaction time would be analogous to the context effects in rating data studied by Anderson (1975) and Parducci (1974) and to the range and regression effects in magnitude scaling studied by $R$. Teghtsoonian and M. Teghtsoonian (1978).

The purpose of the present experiment was to directly compare mental rotation response time functions under

\footnotetext{
I thank Sara C. Hazard, who assisted in running the experiment, and Joseph S. Rossi, who contributed useful suggestions. Please address correspondence to the author at the Department of Psychology, Chafee Social Science Center, University of Rhode Island, Kingston, RI 02881.
}

a skewed distribution and under an even distribution of angular disparities. The experiment also provided an opportunity to replicate the threshold-like nonlinearity, in one or both of these conditions.

\section{METHOD}

\section{Subjects}

Subjects were 20 male and 20 female students at the University of Rhode Island. All had normal or corrected-to-normal visual acuity.

\section{Apparatus and Materials}

One Kodak Ektagraphic random-access slide projector and a Gerbrands tachistoscopic shutter were used to present the stimuli. An Apple II+ computer was programmed to randomize trial sequences and to measure and record response times.

A set of slides was prepared using a pair of Shepard and Metzler's (1971) original block figures. Each slide contained two figures, a standard and a comparison. The standard was either on the left or the right, and was either positive (denoted $\mathrm{P}$ ) or negative (denoted $\mathrm{N}$, and meaning the mirror-image of the positive figure). The orientation of the standard figures was held constant. The comparison figure was either the $\mathrm{P}$ or the $\mathrm{N}$ figure, rotated in the picture plane relative to the standard. The angles of rotation in the even condition were at intervals of $10^{\circ}$ from $0^{\circ}$ to $180^{\circ}$. The angles of rotation in the skewed condition were at intervals of $5^{\circ}$ from $0^{\circ}$ to $60^{\circ}$, and at intervals of $20^{\circ}$ from $60^{\circ}$ to $180^{\circ}$.

\section{Procedure}

Subjects were randomly assigned to either the even or the skewed condition; there were 10 males and 10 females in each condition. Subjects were shown examples of same (P-P and N-N) and different (P-N and N-P) figure pairs, and then completed one session consisting of five blocks of 38 trials each. The first block was considered practice and was discarded from the analysis. The remaining four blocks yielded up to four correct "same" response times at each angular disparity for each subject. Incorrect responses were not rerun. The median of the correct response times was taken as the response time value for that subject and angle. In the 40 (subject) $\times 19$ (angle) data matrix, there was only one case in which all four responses were wrong; an unweighted-means interpolation was made for that case in the trend analyses.

Other aspects of procedure were similar to Rossi and Collyer's (1986) Experiment 1.

\section{RESULTS}

Figure 1 shows response time as a function of angular disparity for the even and skewed conditions. Each point on the graph represents the mean of the 20 median cor- 


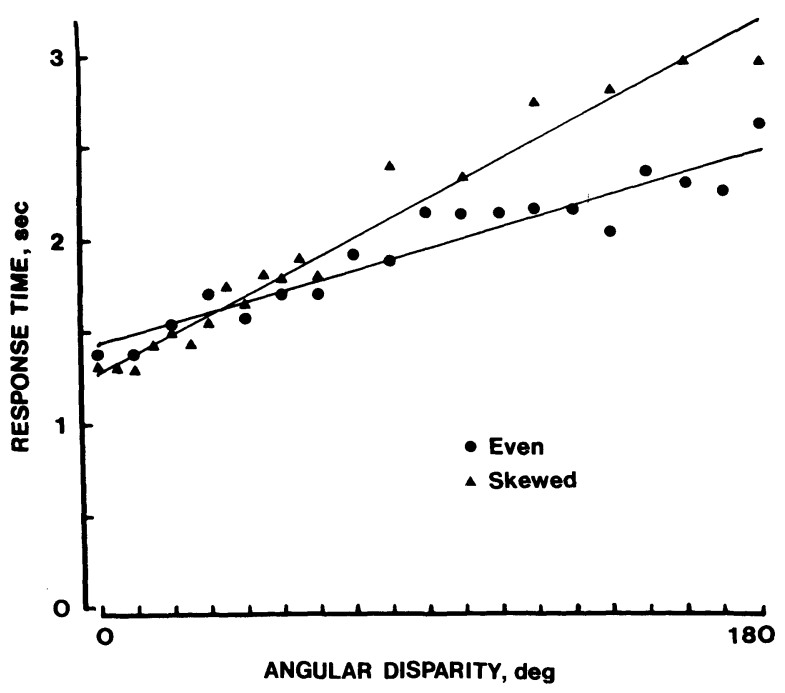

Figure 1. Response time (in seconds) as a function of angular disparity for the even and skewed conditions. The regression lines have significantly different slopes.

rect response times contributed by the participants in that condition. Standard errors of these means range from about $100 \mathrm{msec}$ (both conditions) for small angles to about $200 \mathrm{msec}$ (even) and $300 \mathrm{msec}$ (skewed) for large angles.

Trend analysis of the even condition showed a significant linear component $[F(1,342)=185.83, p<.001]$, accounting for $\omega^{2}=.339$ of the variance. Quadratic and combined higher order trends were not significant. Analysis of the skewed condition, adjusted for the unequal intervals between the angles in this condition, showed a large and significant linear component $[F(1,342)=$ 438.34, $\left.p<.001 ; \omega^{2}=.549\right]$. The quadratic component was also significant $[F(1,342)=5.46, p<.05]$ but small $\left(\omega^{2}=.007\right)$.

Simple regression analyses of the means shown in Figure 1 were used to measure the large linear effects of angular disparity. The regression lines are shown in the figure. In the even condition, the slope was $5.76 \mathrm{msec} /$ degree and the intercept was $1,432 \mathrm{msec}$. In the skewed condition, the slope was $10.49 \mathrm{msec} /$ degree and the intercept was $1,285 \mathrm{msec}$. The difference between the two slopes was significant $[t(34)=6.77, p<.01]$.
This difference is the angle-distribution effect referred to in the title.

\section{DISCUSSION}

The distribution of angular disparities in a mental rotation task affects the slope of the response time function. In this study, skewing the distribution toward the small angles increased the slope of the function. One interpretation of this effect is that the distribution of angles directly affects a parameter, such as the rate of mental rotation, for all angles. Other interpretations may posit differential effects-of practice, for example-on some but not all angles. Further experiments will be needed to explore the alternatives.

Visual inspection of Figure 1 shows considerable overlap between the two conditions for angles less than about $90^{\circ}$. Differences in form between the two conditions are not apparent, in spite of the small quadratic trend found in the skewed condition. It is likely that this trend reflects a pattern in the large-angle data points.

At small angles, visual inspection produces mixed results. On the one hand, it can lead one to perceive that response time is independent of angles below about $15^{\circ}$ in both conditions. This kind of independence was also seen in the Rossi and Collyer (1986) data, and is consistent with the hypothesis of a threshold for mental rotation. On the other hand, the variability of nearby data points discourages any strong conclusion about the detailed form of the response time function.

In conclusion, a distribution of angular disparities skewed toward small angles changes the overall slope of the mental rotation response time function, but does not induce a marked threshold-like departure from linearity. If one sees a threshold in the present data, one must see a threshold in both conditions. However, a good criterion for judging the presence of a threshold is still elusive. If one views Figure 1 with a more conservative eye, the threshold must disappear in both conditions.

Meanwhile, the angle-distribution slope effect remains to be accounted for, and is the subject of further research.

\section{REFERENCES}

ANDERSON, N. H. (1975). On the role of context effects in psychophysical judgment. Psychological Review, 82, 462-482.

Collyer, C. E. (1985). Comparing strong and weak models by fitting them to computer-generated data. Perception \& Psychophysics, 38, 476-481.

PARDUCCI, A. (1974). Contextual effects: A range-frequency analysis. In E. C. Carterette \& M. P. Friedman (Eds.), Handbook of perception (Vol. 2, pp. 127-141). New York: Academic Press.

Rossi, J. S., \& Collyer, C. E. (1986). Is there a threshold for mental rotation? Bulletin of the Psychonomic Society, 24, 1-3.

ShePARD, R. N., \& MeTZler, J. (1971). Mental rotation of threedimensional objects. Science, 171, 701-703.

Teghtsoonian, R., \& Teghtsoonian, M. (1978). Range and regression effects in magnitude scaling. Perception \& Psychophysics, 24, 305-314.

(Manuscript received for publication November 14, 1986.) 